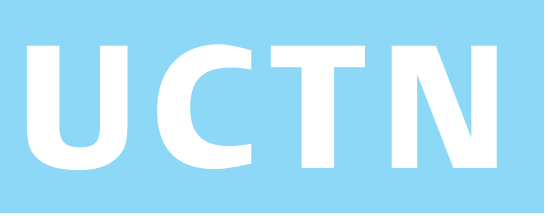

\title{
Gastrointestinal bleeding from gastric metastasis of renal cell carcinoma, treated by endoscopic polypectomy
}

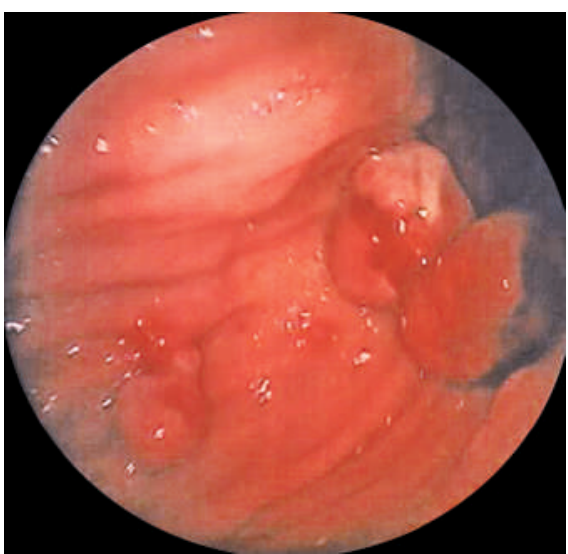

Figure 1 Endoscopic view of bleeding polypoid lesions in the stomach.

Metastatic tumors of the stomach are very rare. We report here a case of successful endoscopic treatment of gastrointestinal hemorrhage from gastric metastases of a renal cell carcinoma (RCC), 5 years after radical resection.

A 78-year-old man was admitted because of anemia. His medical history was noteworthy for radical resection of an RCC 5 years earlier. Upper gastrointestinal endoscopy showed three actively bleeding polypoid lesions of $2-3 \mathrm{~cm}$ within the body of the stomach (Figure $\mathbf{1}$ ). The polyps were removed by electrosurgical snare resection after injection of epinephrine solution in the stalk. No further bleeding was observed after the procedure.

Unexpectedly, at histological examination of the polyps, clear cells were seen, suggesting a metastasis of an RCC (Figure 2). The patient was referred to the oncology department and died 6 months later but he did not present other bleeding episodes during that period.

The incidence of metastatic involvement of the stomach is extremely low $(0.2 \%-$ $0.7 \%$ ) even in autopsy studies $[1-2]$. The majority of the patients described had an ulcer-like bleeding lesion and were treated with embolization [3], with epinephrine injection [4], or surgically [2]; we are not aware of any other report of endo-

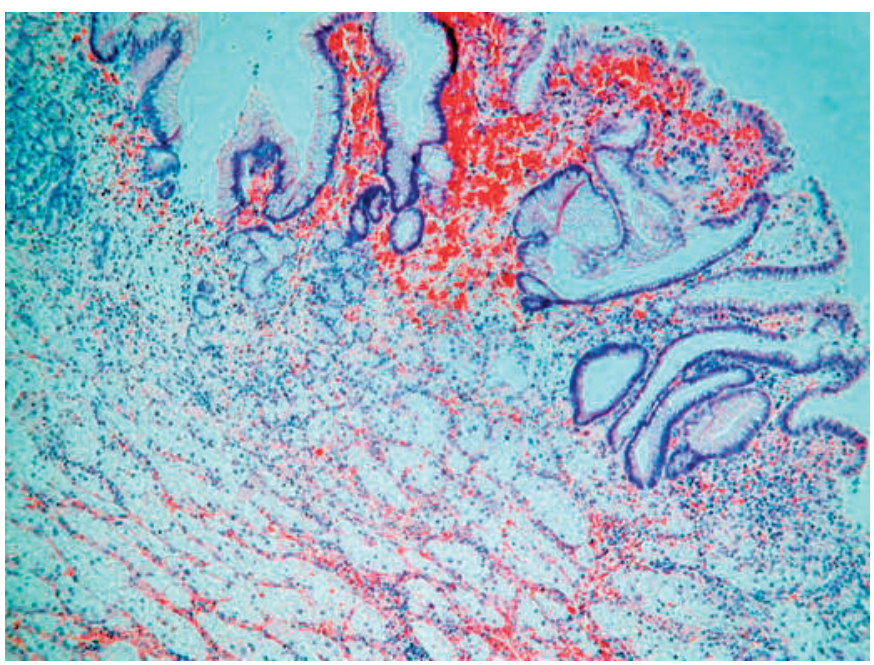

Figure 2 Histological examination showing clear cell kidney carcinoma associated with gastric mucosa (hematoxylin and eosin; $\times 140$ ).

scopic resection of gastric metastases. We performed this procedure because our patient's lesions looked like bleeding polyps and we did not suspect their histological nature. Nevertheless this treatment was effective in stopping the bleeding, and we avoided surgical resection in this patient with disseminated RCC and at high operative risk. For patients with metastatic gastric tumors, surgical resection is still recommended in selected cases as an effective palliation [2-5], but endoscopic therapy of these lesions seems a reasonable option for those patients not eligible for surgery, as was shown in our patient.

Endoscopy_UCTN_Code_CCL_1AB_2AD_3AB

\section{A. Pezzoli, V. Matarese, S. Boccia,}

\section{Simone, S. Gullini}

Department of Gastroenterology and Gastrointestinal Endoscopy, University Hospital, Ferrara, Italy.

\section{References}

${ }^{1}$ Linda K. Hematogenous metastasis to the stomach. Cancer 1990; 65: 1596-1600

${ }^{2}$ Kobayashi O, Murakami H, Yoshida T et al. Clinical diagnosis of metastatic gastric tumors: clinicopathologic findings and prognosis on nine patients in a single cancer center. World J Surg 2004; 28: 548 - 551

${ }^{3}$ Blake M, Owens A, O’Donoghue DP, MacErlean DP. Embolotheraphy for massive upper gastrointestinal haemorrhage secondary to metastatic renal cell carcinoma: report of three cases. Gut 1995; 37: 835-837

4 Picchio M, Paioletti A, Santini E et al. Gastric metastasis from renal cell carcinoma fourteen years after radical nephrectomy. Acta Chir Belg 2000; 100: 228 - 230

${ }^{5}$ Ihde JK, Coit DG. Melanoma metastatic to stomach, small bowel, or colon. Am J Surg 1991; 162: $208-211$

Corresponding author

\section{A. Pezzoli, MD}

Department of Gastroenterology and Gastrointestinal Endoscopy University Hospital Sant'Anna

Corso Giovecca 203

44100 Ferrara

Italy

Fax: $\quad+39-05-32236932$

Email: a.pezzoli@ospfe.it 